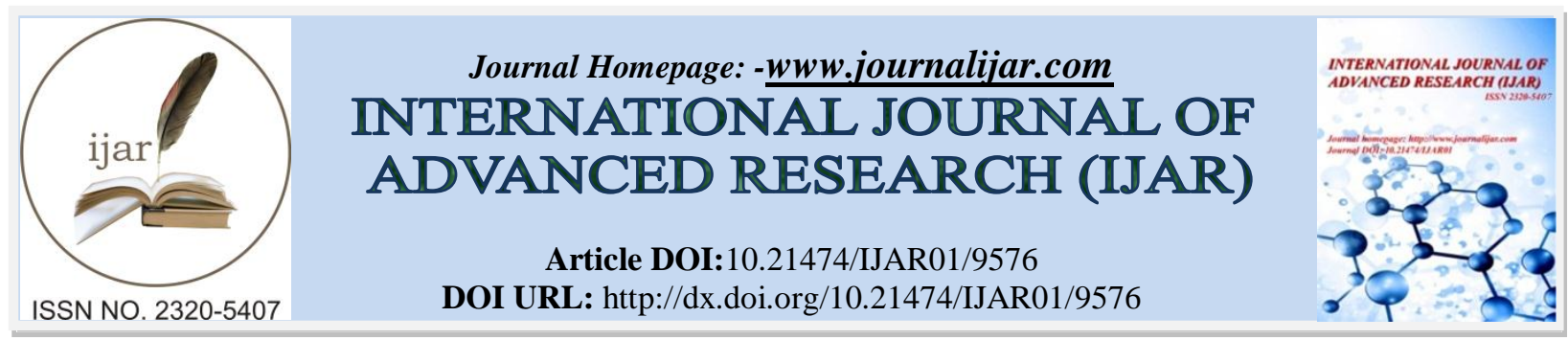

RESEARCH ARTICLE

\title{
LES SEQUELLES DE BRULURES CHEZ L'ENFANT : DONNEES EPIDEMIOLOGIQUES, CLINIQUES ET THERAPEUTIQUES. SEQUELAE OF BURNS IN CHILDREN: EPIDEMIOLOGICAL, CLINICAL AND THERAPEUTIC DATA.
}

\section{D. El amrani ${ }^{1,2}$, A.Bhihi ${ }^{1}$, M. Akenssous ${ }^{1}$ and Y. Benchamkha ${ }^{1}$.}

1. Service de chirurgieplastiqueet reconstructive, CHU Mohamed VI, Marrakech, Maroc. Department of Plastic and Reconstructive Surgery, CHU Mohamed VI, Marrakech, Morocco.

2. Laboratoired'anatomie, Faculté de médecine, Université Cadi Ayyad, Marrakech, Maroc. Laboratory of Anatomy, Medical School, University of Cadi Ayyad, Marrakech, Morocco.

\section{Manuscript Info}

Manuscript History

Received: 14 June 2019

Final Accepted: 16 July 2019

Published: August 2019

Key words:-

Burn-Sequelae-Children.

\section{Abstract}

The sequelae of burns are defined as all the lesions that persist after six months of burn evolution. The purpose of this work is to report the different epidemiological aspects, etiological, clinical as well as the main therapeutic processes used in surgery of children with post-burn sequelae and emphasize the value of prevention. We retrospectively studied 143 patients who underwent surgery for post-burn sequelae and under 15 years. These patients are collected in the plastic surgery and burn department at Mohammed VI University Hospital in Marrakech for a period of 6 years from January 2012 to December 2017. The average age of patients was 6 years and 10 months. The sex ratio was 1,04. Two thirds of the population studied are of low socio-economic status. The causal agent was most often thermal, type boiling liquid $(53,8 \%)$. The circumstances of the initial burn most frequently found were domestic accidents with a rate of $90,9 \%$ of all accidents, predominating in the summer period. Clinically, the sequelae were dominated by the contractures being $80,4 \%$, associated in some cases to other types of sequelae which are: hypertrophic scarring, keloid, dyschromia and scar alopecia. These sequelae were located mainly at the upper limb $(80,41 \%)$, the hand was the predominant site $(39,8 \%)$. Over the 143 patients, 327 surgeries were performed in multiple operating times using different surgical techniques mainly: Z-plasty and derivations, skin graft, excisions-sutures and others taking into account the possibilities of rehabilitation. Results were rated satisfactory in $91 \%$ of our patients. Post-burn sequelae in children are frequent and serious, posing a major therapeutic problem, which gives an importance to the prevention.

Copy Right, IJAR, 2019,. All rights reserved.

\section{Introduction:-}

Un enfant brûlé est avant tout un infirme de la peau ayant subi une destruction plus ou moins profonde de l'enveloppe qui le protège du monde extérieur. Les soins locaux permettent d'aboutir à une cicatrisation initiale mais la vigilance active est impérative[1], pour limiter les séquelles qui se définissent comme l'ensemble des lésions qui 
persistent après six mois d'évolution de la brûlure. Elles se caractérisent par un polymorphisme lésionnel qui englobe séquelles cutanées mineures et majeures, séquelles extra cutanées et séquelles psychologiques[2]. Les séquelles de brûlures sont fréquentes et graves chez l'enfant avec un risque de retentissement sur la croissance d'où l'intérêt d'une réparation précoce qui doit répondre à deux impératifs thérapeutiques :l'un fonctionnel et l'autre esthétique. Le contexte « d'être en croissance » impose une prise en charge adaptée qui ne doit se concevoir qu'avec un environnement de rééducation et de réadaptation[1]. Seule la prévention de la brûlure reste l'arme de choix.Notre étude a pour but d'illustrer les différents aspects épidémiologiques, étiologiques et cliniques des séquelles de brûlures chez l'enfant, ainsi que les principaux procédés thérapeutiques utilisés et leurs résultats.

\section{Patients Et Methodes:-}

Il s'agissait d'une étude rétrospective descriptive d'une série de 143 patients, menée dans le service de chirurgie plastique et brulés au CHU Mohammed VI de Marrakech sur une période de 6 ans, s'étalant entre Janvier 2012 et décembre 2017.

Nous avons inclus les enfants suivis pour séquelles de brûlures ayant été opérés au service et âgé moins de 15 ans.Nous avons exclu de l'étude les patients ayant été vus en consultation mais qui n'ont pas été opérés et les patients à dossiers inexploitables.

Les renseignements recueillis pour la réalisation de cette étude ont été puisés dans les dossiers d'hospitalisation et qui ont été rédigés sur une fiche d'exploitation préétablie.

\section{Resultats:-}

L'âge moyen de nos patients était de 6 ans et 10 mois, avec des extrêmes variant de 7 mois et 15 ans. La tranche d'âge la plus touchée se situait entre 8 ans et 15 ans. Le sexe ratio était de 1,04 objectivant une légère prédominance masculine de 51\%. Concernant Les causes de brûlures, elles étaient multiples. Dans notre série on rapporte : Brûlure par liquide bouillant : 77 cas soit $54 \%$, brûlure par flamme : 40 cas soit $28 \%$, brûlure par braise : 18 cas soit 12\%, cause électrique : 8 cas soit $6 \%$ dont 5 cas étaient victimes de brûlures électriques de haut voltage. Dans notre étude, les accidents domestiques étaient le contexte prédominant de survenue de brûlures chez l'enfant et la cuisine constituait l'endroit où il y avait plus de brûlures, avec une prédominance à la période estivale qui correspondait aussi au mois de Ramadan.

Dans notre série, 122 patients n'avaient aucun antécédent pathologique, chez le reste des patients, les antécédents rapportés étaient : 15 cas d'antécédent de séjour en réanimation suite à la brûlure, 2 cas de sepsis compliquant la brûlure, 2 cas d'épilepsie, 1 cas de trisomie 21 et 1 cas traité par Jebbar.

Ence qui concerne la topographie des séquelles, le membre supérieur étaient les plus fréquentes $80,41 \%$. La main était la partie du corps avec le plus de séquelles chez 57 patients soit $39.8 \%$.

Les brides rétractiles étaient le type de séquelles le plus dominant, rencontré chez $80.4 \%$ des enfants. Nous avons constaté que le tableau clinique se caractérisait par un polymorphisme lésionnel qui varie en fonction de la région anatomique atteinte.

Tous les patients ont bénéficié d'un bilan hématologique : une numération formule sanguine et plaquettes et d'un bilan d'hémostase fait de : taux de prothrombine et temps de céphaline activée. $11.18 \%$ des patients ont bénéficié d'une imagerie radiographique, $7 \%$ des patients ont bénéficié d'un bilan rénal fait de : urée, créatinine et $2.1 \%$ des patients ont bénéficié d'un examen anatomopathologique.

Sur nos 143 patients, 327 interventions chirurgicales ontétéréaliséesenplusieurs temps opératoires, ellesont fait appel à plusieurs techniques, les deux tiers de ces interventions chirurgicales étaient des plasties locales et dont la plastie en $\mathrm{Z}$ était le chef de file. A ce traitement de base ont été associés d'autres gestes selon le siège de la séquelle : Embrochage dans 49 cas et commissuroplastie dans 7 cas.

Le traitement médical et le traitement physique ont fait appel à des infiltrations par des corticoïdes retards chez 11 patients, une immobilisation par attelle ou orthèse selon les cas, une rééducation fonctionnelle chez 11 patients 
comprenant des massages cicatriciels et mise d'attelle de posture, une pressothérapie chez 12 patients, l'application de plaque de silicone chez 2 patients et le port de vêtements compressifs chez 5 patients.

La protection solaire est d'indication systématique au niveau des zones photo exposées. La prise en charge psychologique chez un seul cas.

En ce qui concerne les complications déplorées dans notre étude, nous avons noté 2 cas d'infections de prothèse d'expansion qui a conduit au retrait de la prothèse.

Pour le recul, 120 patients soit $(83.9 \%)$ ont été revus en consultation à l'occasion d'un complément de prise en charge avec un recul moyen de 2 ans et 9 mois (extrêmes allant de 6 mois à 5 ans). Les résultats fonctionnels et esthétiques étaient jugés satisfaisants par rapport au degré de satisfaction subjective du patient et par l'appréciation objective du chirurgien. $91 \%$ de nos patients étaient satisfaits.

\section{Discussion:-}

Sur le plan épidémiologique, l'âge moyen dans notre série était de 6 ans et 10 mois avec des extrêmes de 7 mois et 15 ans. Cette moyenne concorde avec celle de la série Goverman et Al[3]. Quant aux séries d'El Ezzi et Al[4]et Mouhssine[5], la population étudiée était plus jeune, contrairement à l'étude Gupta et Al[6] où l'âge moyen était nettement supérieure atteignant 12 ans et 2 mois.

Notre étude conclut que le sexe masculin est légèrement prédominant avec un pourcentage de $51 \%$, ce qui concorde avec les résultats de la série Gupta et $\mathrm{Al}[6]$. En opposition à la série d'El Ezzi et Al[4] où les filles sont les plus touchées $(56 \%)$. Cette prédominance féminine a été expliquée par le fait que les filles sont plus attirées par le changement de l'image corporelle (esthétique) que les garçons.

Les séries de Mouhssine[5] et Goverman et $\mathrm{Al}[3]$, quant à eux, rapportent une nette prédominance masculine.Dans notre étude, nous avonsconstaté que presque la moitié (53.8\%) des enfants ont été victimes de brûlures thermiques par divers liquides chauds, le thé en était l'élément le plus prépondérant, ceci est expliqué par le fait qu'il représente la boisson chaude la plus répandue au Maroc. La 2ème cause thermique est représentée par la flamme (28\%).Les brûlures électriques représentaient $5.6 \%$ et peuvent survenir lorsque l'enfant met un objet comme une cléou un clou dans une prise murale ou mord dans un erallonge électrique branchée ou encore à l'occasion de l'escalade des poteaux électriques publics.

A l'opposition de nos résultats, les séries de Goverman et Al[3]et Mouhssine[5] rapportent que la lère cause de brûlure est représentée par la flamme suivie de liquides chauds. Les brûlures de cause électrique représentaient 6.6\% dans la série de Goverman et $\mathrm{Al}[3] \mathrm{ce}$ qui corrobore avec notre étude. Quant à Gupta et $\mathrm{Al}[6]$, les brûlures de cause électrique étaient les plus fréquentes dues à la mauvaise qualité de l'installation électrique domestique, suivies des agents thermiques dont les feux d'artifice étaient le chef de file alors que les liquides chauds ne représentaient que $(8,2 \%)$ des causes de brûlures.

Dans notre série, La localisation cervico-céphalique représentait $44.8 \%$ de l'ensemble des séquelles de brûlures chez l'enfant, ce qui concorde avec les données des séries de Mohsine[7]et El Ezzi et Al[4]. Alors que dans les series d'Elamrani et $\mathrm{Al}[8]$ et Mouhssine[5] ce taux est très diminué. Les brides rétractiles étaient le type de séquelles le plus dominant, rencontré chez $80.4 \%$ des enfants dans notre étude, dont la plupart ont présenté en plus, d'autres séquelles à type de cicatrices hypertrophiques et de dyschromies. Notre série est caractérisée par un polymorphisme lésionnel. Les séries Chafiki et Al[2] et Mouhssine[5]rapportent des résultats qui concordent avec notre étude.

Sur nos 143 patients, 327 interventions chirurgicales ont été réalisées. Parmi les techniques chirurgicales utilisées, les plasties locales sont au premier rang avec un nombre de 197. Il s'agit essentiellement de la plastie en Z, en trident, en YV, en IC, enoméga et la plastie en queue de poisson ce qui corrobore avec les séries Sankale et Al[9] et Mouhssine[5]. Alors que les séries Chafiki et Al[10] et El Ezzi et Al[4] ont rapporté un pourcentage diminué dans l'utilisation des plasties locales, par contre les greffes de peau étaient les interventions les plus prédominants dans ces études.

Pour le recul : 83.9\% de nos patients ont été revus en consultation avec un recul moyen de 2 ans et 9 mois (extrêmes de 6 mois et 5ans), ce qui paraît un recul important par rapport à celui de la série Sankale et Al[9] qui est de 5 mois. 
Dans notre série, le recul confirme l'intérêt apporté par le chirurgien dans le but d'assurer un suivi de l'enfant brûlé pendant une bonne période de sa croissance.

Concernant les résultats thérapeutiques, $91 \%$ de nos patients étaient satisfaits des résultats fonctionnels et esthétiques, ce qui rejoint les résultats de l'étude de Mohsine[7].

\section{Conclusion:-}

Les enfantspaientunlourdtribut aux brûlures qui leurlaissent de graves séquellesfonctionnelles, esthétiques et psychologiquesparfoisdramatiques qui font tout l'intérêt de la prévention.

\section{References:-}

1. Poiret Getal., «Le traitement des séquelles de brûlure de l'enfant», /data/revues/02941260/v56i5/S0294126011000926/, oct. 2011.

2. Chafiki N et al. «Les Sequelles de brûlures : Epidemiologie et Traitements », Ann. Burns Fire Disasters, vol. 20, n 3 , p. 129, sept. 2007.

3. Goverman Jet al., « Pediatric Contractures in Burn Injury: A Burn Model System National Database Study », J. Burn Care Res. Off. Publ. Am. Burn Assoc., vol. 38, no 1, p. e192-e199, févr. 2017.

4. El Ezzi O et al.« Surgery on burns sequelae in developing countries », Ann. Burns Fire Disasters, vol. 30, no 1, p. 47-51, mars 2017.

5. Mouhssine M, «Séquelles de brûlureschezl'enfant (à propos de 49 cas avec revue de la littérature) », 2015.

6. Gupta R.K et al.« Neglected post burns contracture of hand in children: Analysis of contributory socio-cultural factors and the impact of neglect on outcome », J. Clin. Orthop. Trauma, vol. 5, no 4, p. 215-220, déc. 2014.

7. Mohsine M.I, « séquelles de brûlures au Maroc: Aspect épidémiologiqueetthérapeutique (à propos de 133 cas). », 2017.

8. Elamrani D et al. «Les retractions cervicales post-brulure - a propos de 49 cas et revue de la litterature », Ann. Burns Fire Disasters, vol. 24, no 3, p. 149-156, sept. 2011.

9. Sankale A.A et al. «Les Cicatrices Retractiles Post- brûlures Du MembreInferieur Chez L'Enfant », Ann. Burns Fire Disasters, vol. 23, no 2, p. 75-80, juin 2010. 